\title{
Editorial
}

\section{Not Everything That Looks like a Lymph Node Is a Lymph Node: A Pitfall for Transbronchial Needle Aspiration}

\author{
Stefano Gasparini \\ Pulmonary Diseases Unit, Department of Internal Medicine, Immunoallergic and Respiratory Diseases, \\ Azienda Ospedaliero-Universitaria 'Ospedali Riuniti', Ancona, Italy
}

Nearly 30 years have passed since the initial experience of Wang et al. [1] on transbronchial needle aspiration (TBNA) was published. Despite the fact that in subsequent years a large number of papers have demonstrated that traditional TBNA is a safe technique, for both diagnostic and staging purposes, several pulmonologists are still reluctant to consider TBNA as a routine procedure to be performed during the first diagnostic bronchoscopy in every bronchoscopic suite. One of the main reasons cited for not performing TBNA is the fear of puncturing vascular structures of the mediastinum. Whenever fellows attending our Bronchoscopic Service ask me about this risk, I always remember an episode that occurred several years ago when I was a student at the Department of Radiology. The first procedure I saw was a renal arteriography performed at that time by injecting the contrast media with a large 17-gauge needle inserted percutaneously through the left lumbar area directly into the abdominal aorta, without any complications or extravascular bleeding. In fact, the risk of clinically relevant bleeding in TBNA is very low even if a large vessel is unintentionally punctured, and just a few cases of major bleeding complications have been reported in the literature [2-4]. However, bronchoscopists must take great care to avoid vascular structures, not only to minimize the risk of complications but also because the puncturing of a vessel will result in a useless and nondiagnostic sample.

Apart from the large vessels, there are two other structures that can be punctured by TBNA; this should be avoided because of possible complications. They are the visceral pleura and the pericardium.

The visceral pleura is in contact with the airways in the right paratracheal area above the azygos arch and at the back of the carina and of the right main bronchus (azygoesophageal recess). The puncture of the visceral pleura may result in a pneumothorax which could occur when a right paratracheal lymph node is approached inserting the needle too laterally (the correct site for sampling the right paratracheal lymph nodes is the 1-2 o'clock position of the 2 nd to 4 th intercartilaginous space of the lower trachea). The sampling of the posterior carina nodes is indicated only when a CT scan shows an enlarged lymph node in that area, because the puncturing of the azygoesophageal recess may also lead to visceral pleural damage and a consequent pneumothorax [5].

The pericardium is also in contact with the airways and can be punctured by TBNA. In effect, this possibility has been deliberately used for diagnostic and therapeutic

\section{KARGER}

Fax +4161306 1234

E-Mail karger@karger.ch

www.karger.com
(C) 2011 S. Karger AG, Basel

0025-7931/11/0821-0001\$38.00/0

Accessible online at:

www.karger.com/res
Stefano Gasparini, MD

SOD Pneumologia

Azienda Ospedaliero-Universitaria 'Ospedali Riuniti'

Via Conca, 71, IT-60020 Ancona (Italy)

Tel. +39071 596 5694, E-Mail s.gasparini@fastnet.it 
purposes to drain pericardial effusion, inserting the needle without complications into the anterior wall of the left lower lobe bronchus or into the anterior wall of the distal trachea, which is in contact with the superior aortic recess of the pericardium [6]. The posterior portion of this pericardial recess lies between the posterior wall of the ascending aorta and the trachea (superior pericardial recess or superior sinus) and it can be recognized on a CT scan in up to $50 \%$ of healthy subjects as a curvilinear, 'halfmoon' or triangular-shaped, near-water-density structure [7]. Sometimes (2-6\% of normal subjects), this recess extends cephalad and rightwards into the right paratracheal region (the so-called 'high-riding' superior pericardial recess) and may mimic right paratracheal lymphoadenopathy or a cystic mediastinal lesion $[8,9]$.

In this issue of Respiration, Gross and Diacon [10] report a case of TBNA performed for staging purposes in a precarinal lesion evaluated as a lymph node in a patient with a left pulmonary nodule suspected for cancer. The procedure was complicated by a hemopericardium with a consequent life-threatening cardiac tamponade, and this is actually the first report of such an occurrence following TBNA. In the discussion of that paper, the authors recognize that the superior pericardial recess was misinterpreted as a lymph node and, in effect, a subsequent surgical staging procedure (mediastinoscopy) did not identify any paratracheal lymph nodal involvement.

The authors must be commended and thanked for publishing this complication and for drawing attention to a possible misinterpretation, thus giving all the pulmonologists who perform TBNA an excellent opportunity to learn. I do not know how many bronchoscopists puncturing the 'high-riding' pericardial recess by TBNA were convinced that they approached a lymph node, but I assume that several colleagues have made the same misinterpretation during their clinical practice. Personally, I remember at least three cases of suspected right paratracheal lymphoadenopathy where I performed a TBNA and, to my surprise, aspirated pericardial fluid. Fortunately, there were no complications. In effect, in the case of Gross and Diacon [10], the hemopericardium was not the result of puncturing the pericardium per se, but, probably, was due to the injury to the back wall of the ascending aorta by the tip of the needle that created a direct communication between the artery and the pericardium. The blood, coming at high pressure from the aorta, did not find any resistance in the low-pressure pericardial cavity and was able to flow smoothly.

Four questions and four consequent lessons can be learnt from this very interesting case report:
(1) Could the pericardial nature of the lesion have been suspected before the CT scan evaluation? The answer is yes. In fact, looking at figure 1 of Gross and Diacon [10], some aspects were present that radiologists have described as useful signs to distinguish between a lymph node and a pericardial recess $[7-9,11]$. The internal homogeneity of the lesion, its 'half-moon' shape, the direct contact with the aorta and the absence of an intervening fat plane between the lesion and the artery are all evident in the CT scan of the reported case. Bronchoscopists should be aware of this anatomical entity and of its imaging characteristics since a 'high-riding' pericardial recess is not rare. I suggest that the readers look very carefully at figure 1 of Gross and Diacon [10] and try to memorize this image since cases like this could happen any time in their clinical practice. If a lesion is suspected to be pericardial in nature, other imaging evaluations, such as the thin section CT, multiplanar reformation or evidence of a change in configuration of this structure on CT scan obtained within a short interval [12] should be considered before performing TBNA.

(2) Could the needle employed have been a factor in determining the complication? As reported by the authors in the discussion, the answer is yes. The authors used the longest needle available for traditional TBNA (15 $\mathrm{mm}$ ). We do not know whether using a shorter needle (13 $\mathrm{mm}$ ) would also have caused the hemopericardium. However, the length of the needle should be taken into consideration when TBNA is planned, and a shorter needle must be used when a vascular structure is present immediately behind a small lesion.

(3) Could the use of endobronchial ultrasound-guided TBNA (EBUS-TBNA) have prevented the complication? Again, the answer is yes. EBUS could have shown a dark lesion consistent with a fluid-filled structure, but this can also be observed in very necrotic lymph nodes and the bronchoscopist would probably have performed the puncture anyway. In effect, the puncturing of an unsuspected 'high-riding' pericardial recess has been reported even with EBUS-TBNA [13]. What probably might have changed with the use of EBUS is that the direct visualization of the needle might have avoided the penetration of its tip up to the vascular structure lying behind.

(4) Is the above-reported case a reason to abandon traditional TBNA and to perform EBUS-TBNA? The answer is no. Traditional TBNA remains a safe technique that can be performed routinely in every bronchoscopic center during the first diagnostic bronchoscopy. The lesson to be learnt from this case is that to perform TBNA safely expensive technology is not necessary, but bron- 
choscopists must be able to interpret CT scans very well. They must also know that not everything that looks like a lymph node is a lymph node. The careful analysis of a CT scan is the first and most important step for performing traditional TBNA. A CT scan evaluation together with a deep knowledge of the mediastinum anatomy makes it possible for the bronchoscopist to always figure out what the structures surrounding the tracheobronchial tree are.

One should not be anxious to perform traditional TBNA: it is not a blind procedure. The CT scan is its eye and, if used in the right way, it always shows what is hidden behind the point of entry of the needle, which minimizes risks and increases diagnostic yield.

\section{References}

$>1$ Wang KP, Brower R, Haponik EF, et al: Flexible transbronchial needle aspiration for staging bronchogenic carcinoma. Chest 1983;84:571-576.

$\checkmark 2$ Kucera RF, Wolfe GK, Perry ME: Hemomediastinum after transbronchial needle aspiration. Chest 1986;90:466.

$>3$ Lazzari Agli L, Trisolini R, Burzi M, et al: Mediastinal hematoma following transbronchial needle aspiration. Chest 2002;122: 1106-1107.

$>4$ Talebian M, Recanatini A, Zuccatosta L, et al: Hemomediastinum as a consequence of transbronchial needle aspiration. J Bronchol 2004;11:178-181.
5 Wang KP: Staging of bronchogenic carcinoma by bronchoscopy. Chest 1994;106:588593.

$\checkmark 6$ Ceron L, Manzato M, Mazzaro F, et al: A new diagnostic and therapeutic approach to pericardial effusion: transbronchial needle aspiration. Chest 2003;123:1753-1758.

7 Glazer HS, Aronberg DJ, Sagel SS: Pitfalls in CT recognition of mediastinal lymphadenopathy. AJR Am J Roentgenol 1985;144: 267-274.

$>8$ Choi YW, McAdams HP, Jeon SC, et al: The 'high riding' superior pericardial recess: CT findings. AJR Am J Roentgenol 2000;175: 1025-1028.

-9 Broderick LS, Brooks GN, Kuhlman JE: Anatomic pitfalls of the heart and pericardium. Radiographics 2005;25:441-453.
10 Gross A, Diacon AH: Cardiac tamponade following transbronchial needle aspiration. Respiration 2011;82:56-59.

11 Kodama F, Fultz PJ, Wandtke JC: Comparing thin-section and thick-section CT of pericardial sinuses and recess. AJR Am J Roentgenol 2003;181:1101-1108.

12 Aribandi M: High riding superior pericardial recess: temporal change can help distinguish from mediastinal pathology. South Med J 2007;100:407-408.

13 Fielding D, Hundloe J, Windsor M, et al: High riding pericardial recess. Findings on EBUS TBNA. J Bronchol 2008;15:182-184. 